\title{
On Humbert Matrix Polynomials of Two Variables
}

\author{
Ghazi S. Khammash ${ }^{1}$, A. Shehata ${ }^{2}$ \\ ${ }^{1}$ Department of Mathematics, Al-Aqsa University, Gaza Strip, Palestine \\ ${ }^{2}$ Department of Mathematics, Faculty of Science, Assiut University, Assiut, Egypt \\ Email: ghazikhamash@yahoo.com,drshehata2006@yahoo.com
}

Received July 13, 2012; revised September 6, 2012; accepted September 14, 2012

\begin{abstract}
In this paper we introduce Humbert matrix polynomials of two variables. Some hypergeometric matrix representations of the Humbert matrix polynomials of two variables, the double generating matrix functions and expansions of the Humbert matrix polynomials of two variables in series of Hermite polynomials are given. Results of Gegenbauer matrix polynomials of two variables follow as particular cases of Humbert matrix polynomials of two variables.
\end{abstract}

Keywords: Humbert Matrix Polynomials of Two Variables; Hypergeometric Matrix Function; Matrix Functional Calculus

\section{Introduction}

The special matrix functions appear in statistics, lie group theory and number theory [1-4] and the matrix polynomials have become more important and some results in the theory of classical orthogonal polynomials have been extended to orthogonal matrix polynomials see for instance [5-9].

If $D_{0}$ is the complex plane cut along the negative, real axis and $\log (z)$ denotes the principal logarithm of $z$ (Saks, S. and A. Zygmund, [10]), then $z^{1 / 2}$ represents $\exp (1 / 2 \log )(z)$ if $A$ is a matrix in $C^{N \times N}$ the set of all the eigenvalues of is denoted by the set of all the eigenvalues of $A$ is denoted by $\sigma(A)$. If $f(z)$ and $g(z)$ are holomorphic functions of the complex variable $z$, which are defined in an open set $\Omega$ of the complex plane, and $A$ is a matrix in $C^{N \times N}$ such that $\sigma(A) \subset \Omega$. Then from the properties of the matrix functional calculus, (Dunford N. and J. Schwartz J. [11]), it follows that $f(A) g(A)=g(A) f(A)$. If $A$ is a matrix with $\sigma(A) \subset D_{0}$, then $A^{1 / 2}=\sqrt{A}$ denotes the a image by $z^{1 / 2}$ of the matrix functional calculus acting on the matrix $A$. we say that $A$ is a positive stable matrix if $\operatorname{Re}(z)>0$ for all $z \in \sigma(A)$.

For any matrix $P$ in $C^{N \times N}$ we will exploit the following relation due to [12]

$$
(1-x)^{-p}=\sum_{n=0}^{\infty} \frac{(p)_{n} x^{n}}{n !},|x|<1
$$

Khammash [12], define the Gegenbauer matrix polynomials of two variables by

$$
C_{n, k}^{A}(x, y)=\sum_{r=0}^{\left[\frac{n}{2}\right]\left[\frac{k}{2}\right]} \frac{(-1)^{r+j}(A)_{n+k-r-j}(2 x)^{n-2 r}(2 y)^{k-2 j}}{r ! j !(n-2 r) !(k-2 j) !}
$$

From which it follows that $C_{n, k}^{A}(x, y)$ is a matrix polynomial in two variables $x$ and $y$ of degree precisely $n$ in $x$ and $k$ in $y$.

Also we recall that if $A(k, n)$ are matrix in $C^{N \times N}$ for $n \geq 0$ and $k \geq 0$ that it follows that (Defez and Jódar [14])

$$
\begin{aligned}
& \sum_{n=0}^{\infty} \sum_{k=0}^{\infty} A(k, n)=\sum_{n=0}^{\infty} \sum_{k=0}^{n} A(k, n-k) \\
& \sum_{n=0}^{\infty} \sum_{k=0}^{\infty} A(k, n)=\sum_{n=0}^{\infty} \sum_{k=0}^{[n / 2]} A(k, n-k)
\end{aligned}
$$

and, for $m$ is a positive integer such that $n>m$, then

$$
\begin{aligned}
& \sum_{n=0}^{\infty} \sum_{k=0}^{\infty} A(k, n)=\sum_{n=0}^{\infty} \sum_{k=0}^{[n / m]} A(k, n-k) \\
& \sum_{n=0}^{\infty} \sum_{k=0}^{n} A(k, n)=\sum_{n=0}^{\infty} \sum_{k=0}^{[n / m]} A(k, n-m k+k)
\end{aligned}
$$

We define Humbert matrix polynomials of two variables and discuss its special cases. Some hypergeometric matrix representations of the Humbert matrix polynomials of two variables, the double generating matrix functions and expansions of the Humbert matrix polynomials of two variables in series of Hermite polynomials are given. Some particular cases are also discussed. 


\section{Definition of Humbert Matrix Polynomials of Two Variables}

Let $A$ be a positive stable matrix in $C^{N \times N}$ for a positive integer $m$, we define Humbert matrix polynomials by

$$
\begin{aligned}
& \left(1-\left(m x t-t^{m}\right)-\left(m y s-s^{m}\right)\right)^{-A} \\
& =\sum_{n=0}^{\infty} \sum_{k=0}^{\infty} P_{n, k, m}(x, y, A) t^{n} s^{k}
\end{aligned}
$$

Now (7) it can be written in the form

$$
\begin{aligned}
& \left(1-\left(m x t-t^{m}\right)-\left(m y s-s^{m}\right)\right)^{-A} \\
& =\sum_{n=0}^{\infty} \sum_{k=0}^{\infty} \frac{(A)_{n+k}\left(m x t-t^{m}\right)^{n}\left(m y s-s^{m}\right)^{k}}{n ! k !}
\end{aligned}
$$

and by (3) and (6) respectively, one gets

$$
\begin{aligned}
= & \sum_{n=0}^{\infty} \sum_{k=0}^{\infty} \sum_{r=0}^{n} \sum_{j=0}^{k} \frac{(A)_{n+k}(m x)^{n-r}(m y)^{k-j}(-1)^{r+j}}{r ! j !(n-r) !(k-j) !} \\
& \times t^{n+(m-1) r} s^{k+(m-1) j} \\
= & \sum_{n=0}^{\infty} \sum_{k=0}^{\infty} \sum_{r=0}^{[n / m][k / m]} \sum_{j=0} \frac{(A)_{(n+k)-(m-l)(r+j)}(-1)^{r+j}}{r ! j !(n-m r) !(k-m j) !} \\
& \times(m x)^{n-m r}(m y)^{k-m j} t^{n} s^{k} \\
\sum_{n=0}^{\infty} & \sum_{k=0}^{\infty} P_{n, k, m}(x, y, A) s^{n} t^{k} \\
= & \sum_{n=0}^{\infty} \sum_{k=0}^{\infty} \sum_{r=0}^{[n / m][k / m]} \sum_{j=0} \frac{(A)_{(n+k)-(m-l)(r+j)}(-1)^{r+j}}{r ! j !(n-m r) !(k-m j) !} \\
& \times(m x)^{n-r}(m y)^{k-j} t^{n} s^{k}
\end{aligned}
$$

By equating the coefficients of $t^{n} s^{k}$ in (7) and (8), we obtain an explicit representation of the Humbert matrix polynomials of two variables. In the form

$$
=\sum_{r=0}^{[n / m][k / m]} \sum_{j=0} \frac{(A)_{(n+k)-(m-l)(r+j)}(-1)^{r+j} \times(m x)^{n-m r}(m y)^{k-m j}}{r ! j !(n-m r) !(k-m j) !}
$$

from which it follows that $P_{n, k, m}(x, y, A)$ is a matrix polynomial in two variables $x$ and $y$ of degree precisely $n$ in $x$ and $k$ in $y$. In (9) setting $m=2$, we get the Gegenbauer matrix polynomials of two variables [13] as particular case of the Humbert matrix polynomials of two variables.

\section{Hypergeometric Matrix Representation for $P_{n, k, m}(x, y, A)$}

We study here the representation of the hypergeometric matrix representation for the Humbert matrix polynomials of two variables. There are some facts and notations used throughout the development in Sections 3 - 5, which are listed here.

Fact 1. [15] The reciprocal scalar Gamma Function $\Gamma^{-1}(z)=1 / \Gamma(z)$, is an entire functions of the complex variable $z$. Thus, for $c \in C^{N \times N}$, the Riesz-Dunford functional calculus [11] shows that $\Gamma^{-1}(z)$ is well defined and is indeed, the inverse of $\Gamma(z)$, Hence: if $c \in C^{r \times r}$ is such that $C+n I$ is invertible for every integer $n \geq 0$. Then

$$
(c)_{n}=\Gamma(c+n I) \Gamma^{-1}(z) .
$$

Fact 2. [12] If $A, B$ and $C$ are members of $C^{r \times r}$ for which $C+n I$ is invertible for every integer $n \geq 0$. The hypergeometric matrix function $F(A, B ; C ; z)$ is defined by

$$
F(A, B ; C ; z)=\sum_{n=0}^{\infty} \frac{1}{n !}\left((A)_{n}(B)_{n}\left[(C)_{n}\right]^{-1}\right) z^{n}
$$

it converges for $|z|<1$.

Notation 1. [16] For $A \in C^{r \times r}$, the matrix version of the pochhammer symbol (the shifted factorial) is

$$
\begin{gathered}
(A)_{n}=A(A+I)(A+2 I) \cdots(A+(n-1) I), \\
n \geq 1
\end{gathered}
$$

with $(A)_{0}=I$.

Note that $A=-j I$, where $j$ is a positive integer, then $(A)_{n}=0$ when ever $n>j$. Also, the product in (10) is commutative, and then it is easy to see that

$$
\begin{gathered}
(A)_{n+k}(A)_{n}(A+n I)_{k} \\
(A)_{n-k}=(-1)^{k}(A)_{n}[(I-A-n I)]^{k-1}
\end{gathered}
$$

and

$$
(A)_{m n}=m^{m} \prod_{s=1}^{m}\left(\frac{1}{m}(A+(s-1)) I\right)_{n}
$$

where $m$ is a positive integer.

Notation 2. [17]

$$
\begin{gathered}
\frac{1}{(n-m k)}=\frac{(-1)^{m k}}{n !}(-n)_{m} k ; 0 \leq m k \leq n \\
(-n I)_{m k}=m^{m k} \prod_{s=1}^{m}\left(\frac{1}{m}(s-n-1) I\right)_{k}
\end{gathered}
$$

Now, in view of Notation 2, the explicit representation (9) for $m \geq 2$, becomes 


$$
\begin{aligned}
P_{n, k, m}(x, y, A)= & \sum_{r=0}^{\left[\frac{n}{m}\right.} \sum_{j=0}^{\left.\frac{k}{m}\right]} \frac{(A)_{(n+k)-(m-l)(r+j)}}{r ! j ! n ! k !} \times(-1)^{(m+l)(r+j)}(m x)^{n-m r}(m y)^{k-m j} \\
& \times m^{m r} \prod_{s=1}^{m}\left(\frac{1}{m}(s-n-1) I\right)_{r} m^{m j} \prod_{l=1}^{m}\left(\frac{1}{m}(l-k-1) I\right)_{j} \\
= & \frac{(A)_{n+k}(m x)^{n}(m y)^{k}}{n ! k !} \times \sum_{r=0}^{\left.\left[\frac{n}{m}\right] \frac{k}{m}\right]} \frac{(1)}{r ! j !}\left[\prod_{s=1}^{m-1}\left(\frac{(-A-(n+k) I+s I)}{(m-1)}\right)_{r+j}\right]^{-1} \\
& \times \prod_{s=1}^{m}\left(\frac{s-n-1}{m} I\right) \prod_{l=1}^{m}\left(\frac{l-k-1}{m} I\right) \times\left(\frac{1}{(m-1)^{m-1} x^{m}}\right)^{r}\left(\frac{1}{(m-1)^{m-1} y^{m}}\right)^{j}
\end{aligned}
$$

Thus we get the following hypergeometric representation of Humbert matrix polynomials of two variables.

$$
\begin{aligned}
P_{n, k, m}(x, y, A)= & \frac{(A)_{n+k}(m x)^{n}(m y)^{k}}{n ! k !} \times{ }_{m} F_{m-1}^{(2)}\left[\frac{-n}{m} I, \frac{n-1}{m} I, \cdots, \frac{n-m+1}{m} I ; \frac{-k}{m} I, \frac{k-1}{m} I, \cdots, \frac{k-m+1}{m} I\right. \\
& \left.\frac{-A-((n+k)-1) I}{m-1}, \frac{-A-((n+k)-2) I}{m-1}, \cdots, \frac{-A-((n+k)-(m-1)) I}{m-1} ; \frac{1}{(m-1)^{m-1} x^{m}}, \frac{1}{(m-1)^{m-1} y^{m}}\right]
\end{aligned}
$$

For $m=2$ (11), we gives hypergeometric representation of Gegenbauer matrix polynomials of two variables [13].

The above facts and notations will be used throughout the next two sections.

\section{Additional Double Generating Matrix Functions}

Now, since

$$
\begin{aligned}
P_{n, k, m}(x, y, A) & =\sum_{r=0}^{\left[\frac{n}{m}\right]} \sum_{j=0}^{\left.\frac{k}{m}\right]} \frac{(A)_{(n+k)-(m-l)(r+j)}}{r ! j !(n-m r) !(k-m j) !} \times(-1)^{r+j}(m x)^{n-r}(m y)^{k-j} \sum_{n=0 k=0}^{\infty} \sum_{n, k, m}^{\infty}(x, y, A) s^{n} t^{k} \\
& =\sum_{n=0}^{\infty} \sum_{k=0}^{\infty} \sum_{r=0}^{\left[\frac{n}{m}\right]} \sum_{j=0}^{\left.\frac{k}{m}\right]} \frac{(A)_{(n+k)-(m-l)(r+j)}}{(A)_{n+k} r ! j !(n-m r) !(k-m j) !} \times(-1)^{r+j}(m x)^{n-r}(m y)^{k-j} t^{k+m j} s^{n+m r}
\end{aligned}
$$

By using (5), one gets

$$
=\sum_{n=0}^{\infty} \sum_{k=0}^{\infty} \sum_{r=0}^{\infty} \sum_{j=0}^{\infty} \frac{(A)_{(n+k)+(r+j)}}{(A)_{(n+m r)+(k+m)} r ! j ! n ! k !} \times(-1)^{r+j}(m x)^{n-r}(m y)^{k-j} t^{k+m j} s^{n+m r}
$$

By using Notation 1, the following generating matrix functions for Humbert matrix polynomials of two variables follows

$$
\begin{aligned}
& =\sum_{n=0}^{\infty} \sum_{k=0}^{\infty} \sum_{r=0}^{\infty} \sum_{j=0}^{\infty} \frac{(A+(n+k) I)_{(r+j)}}{(A+(n+k) I)_{m(r+j)} r ! j ! n ! k !} \times(-1)^{r+j}(m x)^{n-r}(m y)^{k-j} t^{k+m j} s^{n+m r} \\
& =\sum_{n=0}^{\infty} \sum_{k=0}^{\infty} \sum_{=0}^{\infty} \sum_{j=0}^{\infty} \frac{(A+(n+k) I)_{(r+j)}}{r ! j ! n ! k ! m^{m} \prod_{s=1}^{m}\left(\frac{1}{m}\left(A_{A_{(n+k)}}+(s+1) I\right)\right)_{(r+j)}} \times(-1)^{r+j}(m x)^{n-r}(m y)^{k-j} t^{k+m j} s^{n+m r} \\
& \sum_{n=0}^{\infty} \sum_{k=0}^{\infty} P_{n, k, m}(x, y, A)\left[(A)_{n+k}\right]^{-1} s^{n} t^{k} \\
& =\sum_{n=0}^{\infty} \sum_{k=0}^{\infty} \frac{(m x t)^{n}(m y s)^{k}}{n ! k !} \sum_{r=0}^{\infty} \sum_{j=0}^{\infty} \frac{(A+(n+k) I)_{(r+j)} t^{k+m j} s_{s=1}^{n+m r}\left(\frac{1}{m}\left(A_{(n+k)}+(s+1) I\right)\right)_{(r+j)} r ! j !}{} \times\left(\left(\frac{-s}{m}\right)^{m}\right)^{r}\left(\left(\frac{-t}{m}\right)^{m}\right)^{j}
\end{aligned}
$$


have thus discovered the family of double generating function of the Humbert polynomials of two variables

$$
\begin{aligned}
& \sum_{n=0}^{\infty} \sum_{k=0}^{\infty} P_{n, k, m}(x, y, A)\left[(A)_{n+k}\right]^{-1} s^{n} t^{k}=\sum_{n=0}^{\infty} \sum_{k=0}^{\infty} \frac{(m x t)^{n}(m y s)^{k}}{n ! k !} \\
& \times{ }_{1} F_{m}^{(2)}\left[(A+(n+k) I) ; \frac{A+(n+k) I}{m} I, \frac{A+((n+k)+1) I}{m} I, \cdots, \frac{A+((n+k)+(m-1)) I}{m} I ;\left(\frac{-s}{m}\right)^{m},\left(\frac{-t}{m}\right)^{m}\right]
\end{aligned}
$$

If $B$ is a positive stable matrix in $C^{N \times N}$, then let us now return to (12) and consider the double sum.

$$
\sum_{n=0}^{\infty} \sum_{k=0}^{\infty} \frac{(B)_{n+k} P_{n, k, m}(x, y, A) t^{k} s^{n}}{(A)_{n+k}}=\sum_{n=0}^{\infty} \sum_{k=0}^{\infty} \sum_{r=0}^{\left[\frac{n}{m}\right]} \sum_{j=0}^{\left.\frac{k}{m}\right]} \frac{(B)_{n+k}(A)_{(n+k)-(m-l)(r+j)}}{(A)_{n+k} r ! j !(n-m r) !(k-m j) !} \times(-1)^{r+j}(m x)^{n-r}(m y)^{k-j} t^{k} s^{n}
$$

Then in similar manner, we get

$$
\begin{aligned}
& \sum_{n=0}^{\infty} \sum_{k=0}^{\infty}(B)_{n+k} P_{n, k, m}(x, y, A)\left[(A)_{n+k}\right]^{-1} t^{k} s^{n} \\
= & \sum_{n=0}^{\infty} \sum_{k=0}^{\infty} \frac{(m x t)^{n}(m y s)^{k}}{n ! k !} \times_{m} F_{m-1}^{(2)}\left[\frac{B+(n+k) I}{m} ; \frac{B+((n+k)+1) I}{m}, \frac{B+((n+k)+(m-1)) I}{m},\right. \\
& \left.A+(n+k) I ; \frac{A+(n+k) I}{m} ; \frac{A+((n+k)+1) I}{m}, \frac{A+((n+k)+(m-1)) I}{m} ;-s^{m},-t^{m}\right]
\end{aligned}
$$

\section{Expansions of $P_{n, k, m}(x, y, A)$ in Series of Hermite $H_{n, k}(x, y, A)$}

Now, we derive expansions of $P_{n, k, m}(x, y, A)$ in series of Hermite $H_{n, k}(x, y, A)$ According to [18], the expansion of $x^{n} I$ in a series of Hermite matrix polynomials was given in the form:

$$
\frac{x^{n}}{n !} I=(\sqrt{2 A})^{-n} \sum_{k=0}^{[n / 2]} \frac{y^{k}}{k !(n-2 k) !} H_{n-2 k}(x, y, A)
$$

which with the aid of (5) and (9), one gets

$$
\begin{aligned}
\sum_{n=0}^{\infty} \sum_{k=0}^{\infty} P_{n, k, m}(x, y, A) t^{k} s^{n} & =\sum_{n=0}^{\infty} \sum_{k=0}^{\infty} \sum_{r=0}^{\left[\frac{n}{m}\right]} \sum_{j=0}^{\left.\frac{k}{m}\right]} \frac{(A)_{(n+k)-(m-l)(r+j)}}{r ! j !(n-m r) !(k-m j) !} \times(-1)^{r+j}(m x)^{n-m r}(m y)^{k-m j} t^{k} s^{n} \\
& =\sum_{n=0}^{\infty} \sum_{k=0}^{\infty} \sum_{r=0}^{\infty} \sum_{j=0}^{\infty} \frac{(-1)^{r+j}(A)_{(n+k)(r+j)} \times(m x)^{n}(m y)^{k} t^{k+m j} s^{n+m r}}{r ! j ! n ! k !}
\end{aligned}
$$

From (16), we get

$$
\begin{aligned}
& =\sum_{n=0}^{\infty} \sum_{k=0}^{\infty} \sum_{r=0}^{\infty} \sum_{j=0}^{\infty} \sum_{p=0}^{[n / 2]} \frac{y^{p}(\sqrt{2 A})^{-n}(A)_{(n+k)(r+j)}}{r ! j ! k ! p !(n-2 p) !} \times(-1)^{r+j}(m y)^{k} H_{n-2 p}(m x, y, A) s^{n+m r} t^{k+m j} \\
& =\sum_{n=0}^{\infty} \sum_{k=0}^{\infty} \sum_{r=0}^{\infty} \sum_{j=0}^{\infty} \sum_{p=0}^{\infty} \frac{y^{p}(\sqrt{2 A})^{-n-2 p}(A)_{((n+2 p)+k)+(r+j)}}{r ! j ! k ! p ! n !} \times(-1)^{r+j}(m y)^{k} H_{n}(m x, y, A) s^{n+2 p+m r} t^{k+m j} \\
& =\sum_{n=0}^{\infty} \sum_{k=0}^{\infty} \sum_{r=0}^{\left[\frac{n}{m}\right]} \sum_{j=0}^{\left.\frac{k}{m}\right]} \sum_{p=0}^{\min (r, j)} \frac{(-1)^{(r-p)+(j-p)} y^{p}}{(r-p) !(j-p) !} \times \frac{(\sqrt{2 A})^{-n-2 m-2 p}(m y)^{k-m j}}{(k-m j) !(n-m r) ! p !} \times(A)_{(n+k)-(m-1)(r+j)} H_{n-m r}(m x, y, A) s^{n+(2-m) p} t^{k}
\end{aligned}
$$

Now replacing $n$ by $n+(m-2) p$ and equating the coefficients of $s^{n} t^{k}$, we get

$$
\begin{aligned}
P_{n, k, m}(x, y, A)= & \sum_{r=0}^{[n+(m-2) p / m]} \sum_{j=0}^{\left.\frac{k}{m}\right]} \sum_{p=0}^{\min (r, j)} \frac{(-r)_{p}(-j)_{p}}{r ! j ! p !(k-m j) !} \times \frac{(-1)^{(r+j)}(m)^{k-m j}(y)^{k+p-m j}}{(n-2 p-m(p+r)) !} \\
& \times(\sqrt{2 A})^{-n-m(p+r)}(A)_{(n+k)+(m-2 p)-(m-l)(r+j)} \times H_{n-2 p-m(r+p)}(m x, y, A)
\end{aligned}
$$




\section{REFERENCES}

[1] A. G. Constantine and R. J. Mairhead, "Partial Differential Equations for Hypergeometric Functions of Two Argument Matrices," Journal of Multivariate Analysis, Vol. 2, No. 3, 1972, pp. 332-338. doi:10.1016/0047-259X(72)90020-6.

[2] R. J. Muirhead, "Systems of Partial Differential Equations for Hypergeometric Functions of Matrix Argument," Annals of Mathematical Statistics, Vol. 40, No. 3, 1970, pp. 991-1001. doi:10.1214/aoms/1177696975

[3] A. Terras, "Special Functions the Symmetric Space of Positive Matrices,” SIAM Journal on Mathematical Analysis, Vol. 16, No. 3, 1985, pp. 620-640. doi:10.1137/0516046

[4] A. T. James, "Special Functions of Matrix and Single Argument in Statistics in Theory and Application of Special Functions,” Academic Press, New York, 1975.

[5] A. J. Duran, "Markov's Theorem for Orthogonal Matrix Polynomials," Canadian Journal of Mathematics, Vol. 48, 1996, pp. 1180-1195. doi:10.4153/CJM-1996-062-4

[6] A. J. Duran and W. Van Assche, "Orthogonal Matrix Polynomials and Higher Order Recurrence Relations,” Linear Algebra and Its Applications, Vol. 219, No. 1, 1995, pp. 261-280. doi:10.1016/0024-3795(93)00218-O.

[7] L. Jódar and J. Sastre, “The Growth of Laguerre Matrix Polynomials on Bounded Inervals," Applied Mathematics Letters, Vol. 13, No. 8, 2000, pp. 21- 26. doi:10.1016/S0893-9659(00)00090-2.

[8] L. Jódar, R. Company and E. Navarro, "Laguerre Matrix Polynomials and Systems of Second Order Differential Equations,” Applied Numerical Mathematics, Vol. 15, No. 1, 1994, pp. 53-63. doi:10.1016/0168-9274(94)00012-3.

[9] A. Sinap and W. Van Assch, "Orthogonal Matrix Poly- nomials and Applications,” Journal of Computational and Applied Mathematics, Vol. 66, No. 1-2, 1996, pp. 27-52. doi:10.1016/0377-0427(95)00193-X.

[10] S. Saks and A. Zygmund, “Analytic Functions,” Elsevier, Amsterdam, 1971.

[11] N. Dunford and J. Schwartz, "Linear Operators, Part I," Interscience, New York, 1955.

[12] L. Jódar and J. C. Cortés, “On the Hypergeometric Matrix Function," Journal of Computational and Applied Mathematics, Vol. 99, No. 1-2, 1998, pp. 205-217. doi:10.1016/S0377-0427(98)00158-7

[13] G. S. Khammash, “A Study of a Two Variables Gegenbauer Matrix Polynomials and Second Order Partial Matrix Differential Equations,” International Journal of Mathematical Analysis, Vol. 2, No. 17, 2008, pp. 807-821.

[14] E. Defez and L. Jódar, "Some Applications of the Hermite Matrix Polynomials Series Expansions,” Journal of Computational and Applied Mathematics, Vol. 99, No. 1-2, 1998, pp. 105-117. doi:10.1016/S0377-0427(98)00149-6.

[15] E. Hille, "Lectures on Ordinary Differential Equations," Addison-Wesley, New York, 1969.

[16] L. Jódar and J. C. Cortés, "Some Properties of Gamma and Beta Matrix Functions,” Applied Mathematics Letters, Vol. 11, No. 1, 1998, pp. 89-93. doi:10.1016/S0893-9659(97)00139-0

[17] E. D. Rainvlle, "Special Functions," Chelsea Publishing Co., Bronx, New York, 1960.

[18] R. S. Batahan, "Anew Extension of Hermite Matrix Polynomials and Its Applications,” Linear Algebra and Its Applications, Vol. 419, No. 1, 2006, pp. 82-92. doi:10.1016/j.laa.2006.04.006 